\title{
Application of palliative ventilation: potential and clinical evidence in palliative care
}

\author{
Sebastiano Mercadante ${ }^{1,2}$ - Antonello Giarratano ${ }^{1,2}$ - Andrea Cortegiani ${ }^{1,2}$. \\ Cesare Gregoretti ${ }^{2}$
}

Received: 2 February 2017 / Accepted: 10 April 2017 / Published online: 25 April 2017

(C) Springer-Verlag Berlin Heidelberg 2017

\section{Introduction}

Dyspnea is an unpleasant sensation of breathing, characterized by qualitatively distinct sensations presenting with variable intensity [1]. It may be caused by cancer itself, and preexisting or concomitant co-morbid conditions [2]. Dyspnea is associated with fatigue, anxiety, decreased function and quality of life and increased mortality [3, 4], and significant suffering both for patients and caregivers. This symptom tends to further increase at the lowest levels of functional status, particularly in the last week of life [5]. Dyspnea was the most frequent reason for emergency admission of home care patients [6], where then they died [7] and one of the most frequent indications for palliative sedation [8].

Current management of dyspnea requires the treatment of any reversible cause and supportive measures to minimize the sensation of dyspnea. Opioids may help to relief the burden due to dyspnea when acute respiratory distress is not yet present, although there is some low-quality evidence showing benefits with the use of oral or parenteral opioids to palliate breathlessness [9]. While low flow oxygen has been shown to be efficacious for dyspnea only in hypoxemic patients [10]. The real issue is that many patient still experience persistent

Sebastiano Mercadante

terapiadeldolore@lamaddalenanet.it; 03sebelle@gmail.com

1 Anesthesia and Intensive Care and Pain Relief and Supportive/ Palliative Care, La Maddalena Cancer Center, Via san Lorenzo 312, 90145 Palermo, Italy

2 Department of Biopathology and Medical Biotechnologies (DIBIMED), Section of Anaesthesia, Analgesia, Intensive Care and Emergency, University Hospital Paolo Giaccone, University of Palermo, Palermo, Italy dyspnea despite these interventions, particularly when dyspnea may result in an overt respiratory failure [11].

\section{The concept of palliative ventilation}

New therapies, in particular for the treatment of infections (e.g. new antibiotics and anti-viral agents), new diagnostic procedures (e.g. diffusion of protected bronchoalveolar lavage), new modalities of ventilation, the development of clinical guidelines and better monitoring systems have produced better outcomes in the last years. One of the best examples of the changes in outcomes observed in the last decades was reported in cancer patients admitted to an intensive care unit (ICU) for acute respiratory failure in different periods [12]. Differently from traditional invasive mechanical ventilation, non-invasive ventilation (NIV) does not impair patient's upper airway and glottis function. NIV is able to reduce the work of breathing and to improve gas exchange while preserving the ability to cough, swallow and speak [13]. Furthermore, NIV avoids iatrogenic complications associated with endotracheal intubation and reduces the risk of ventilator-associated pneumonia [14]. In recent years, NIV has been found to have a wide application outside ICU and in home care [15-19]. NIV in general wards has also been used in 'not to intubate' patients. Interestingly, approximately $50 \%$ of patients survived acute events [12, 20]. NIV includes both noninvasive positive pressure ventilation (NPPV) and continuous positive airway pressure (CPAP). During CPAP, the flow and volume are only generated by the patient's respiratory muscles. During NIV, the inspiratory flow is variably generated by the respiratory muscles and the pressure applied by the ventilator (partial support) or completely generated by the ventilator (total support). In patients with terminal disease, NIV is aimed at relieving dyspnea, similarly to pain, or as a therapeutic option to 
allow to save time and gather consent to diagnostic or therapeutic procedures, or simply communicate the short prognosis when abruptly passing from an intensive to a palliative care setting $[16,21]$. In a strictly palliative setting where the main goal is to manage severe respiratory distress in a patient near to death [22], CPAP alone does not have theoretically any role in relieving symptoms, because it does not supply respiratory muscles as NIV.

High-flow nasal therapy (HFNT), a device that delivers high flows of fully humidified inspired gas $\left(37^{\circ} \mathrm{C}, 100 \%\right.$ $\mathrm{RH}, 44 \mathrm{mg} \mathrm{H}_{2} \mathrm{O} / \mathrm{L}$ ) driven by a turbine up to $60 \mathrm{~L} / \mathrm{min}$, by dedicated nasal prongs, has been commercially available [23]. The use of HFNT finds its rationale as 'stand alone' therapy or in patients who cannot tolerate NIV, offering an interface that is less traumatic in term of skin breakdown and that potentially offers less burden for the caregiver. This would make HFNT also suitable as a first-line therapy to relieve severe dyspnea in many conditions [24]. HFNT has also been used in 'do not to intubate' patients in ICU [25]. The mechanisms of action of HFNT include enhanced $\mathrm{O}_{2}$ pharyngeal concentration, enhanced lung mucociliary clearance and humidification, improving patients' comfort and increasing the performance at high respiratory rates. HFNT also reduces the metabolic expenditure of gas conditioning occurring during normal respiration, thus impacting $\mathrm{CO}_{2}$ production [26-28]. HFNT may attenuate the inspiratory resistances by splinting nasopharyngeal boundaries, providing adequate flow, supplying adequately warmed and humidified gases and triggering nasal muscle activation, thereby stiffening the airway. By using HFNT, the pressure at onset of inspiration remains above atmospheric pressure during a large part of the inspiratory phase. This increase would improve the driving pressure for inspiration [29]. Moreover, it has been shown that HFNT may increase expiratory resistance, by a jet-flow effect that creates a pressure gradient across the flow-restricted nose segment with a mechanism that differs from traditional CPAP. Nasal cannula size is a critical factor in determining expiratory positive airway pressure (EPAP) generation during HFNT, as EPAP relates to air leak around the cannula prongs. A washout of nasopharyngeal dead space is consequent to application of HFNT [30]. The differences with CPAP are evident: HFNT not only increases expiratory resistances and decreases the inspiratory resistances in a completely different way of CPAP but also leads to an amelioration of dyspnea by reducing the work of breathing due less need for increasing alveolar ventilation.

\section{Clinical studies of palliative ventilation}

Palliative ventilation represents a field of great potential interest because a subgroup of patients with dyspnea who may develop acute failure of a specific organ that is not necessarily related to the site or progression of the cancer. For example, a consistent part of cancer patients have chronic pulmonary disease or cardiac disease and the occurrence of an acute exacerbation of these diseases, leading to acute respiratory failure, is relatively common. Despite the fact that most of these episodes may be promptly reversible, if adequately treated, these patients sometimes do not receive any form of ventilator support, just because they have an underlying cancer [12].

There are many suggestions that NIV might be an alternative option to relieve dyspnea even in very advanced patients [31]. This population is potentially the ideal candidate for NIV, as most of them are patients with a 'do-not-intubate' code. Indeed, many patients do not receive endotracheal intubation because of the low survival and the burden of an ICU admission.

NIV was used for the first time in a large group of advanced cancer patients with acute respiratory failure who had been judged by the referring oncologist to be suitable only for palliative care [32]. Major criteria for enrolment into the study were a $\mathrm{PaO}_{2} / \mathrm{FiO}_{2}$ ratio $<25$, a $\mathrm{pH}<7.35$ with a partial arterial $\mathrm{CO}_{2}$ tension $\left(\mathrm{PaCO}_{2}\right)>50 \mathrm{mmHg}$, involvement of accessory muscles of respiration or paradoxical breathing with patient reporting subjective distress and/or dyspnea. The duration of NIV was about 6 days. Most of the patients were ventilated in 'protected' environments, unless four who received the treatment directly in the Palliative Care Unit. NIV was discontinued in ten patients, due to intolerance; progressive worsening of blood gas-analysis, $\mathrm{PaO}_{2}$ or $\mathrm{pH}$; sudden death; vomiting; or need to sedate for pain. Two patients were intubated, three received NIV 'ad libitum' and the others were treated only with supportive therapy. The use of NIV improved the immediate outcome in patients who were unlikely to receive any other form of ventilator support, but did not produce any advantage on long-term survival. However, NIV was feasible and useful in quickly improving dyspnea in end-stage cancers patients with respiratory failure.

Subsequently, a randomized-controlled study was performed in patients with end-stage cancer presenting acute respiratory failure and severe distress [33]. Patients were randomized according to their hypercapnic status to oxygen or NIV, and subcutaneous morphine was given as needed. Dyspnea decreased more rapidly in the NIV group with most benefit reported after the first hour of treatment and in hypercapnic patients. Globally, $\mathrm{PaO}_{2}, \mathrm{PaCO}_{2}, \mathrm{pH}$ and respiratory rate were better in hypercapnic patients who received NIV in comparison with those on oxygen. A lower morphine consumption during the first $48 \mathrm{~h}$ was also observed in the NIV group. Of interest, the number of deaths in hypercapnic patients was smaller in the NIV group. NIV was well accepted. This short-term study suggested that NIV was more effective compared to oxygen in reducing dyspnea and decreasing the doses of morphine in patients with end-stage cancer. Sleep problems may arise and some patients may be disturbed by application of NIV. High-flow nasal cannula (HFNC) may 
potentially improve the quality of sleep, also allowing cough, speaking and eating, although no solid data have been published on these aspects.

Few studies have assessed HFNC in advanced cancer patients with hypoxia. HFNC in do not intubate (DNI) patients with hypoxemic respiratory distress provided adequate oxygenation and a decrease of breathing frequency. Only $18 \%$ of patients escalated to NIV and mortality was $60 \%$ suggesting that this mode of ventilation may be an alternative to NIV for DNI patients [34]. In a series of 183 cancer patients, $72 \%$ were given HFNT in the ICU because of hypoxia. Patients improved (41\%) or remained stable $(44 \%)$ while on the device, whereas $15 \%$ declined. Forty-five percent of patients were living, and 55\% had died. The median time on HFNT was 3 days (range 1-27). A do not resuscitate (DNR) order was present in 101 (55\%) patients, either before $(12 \%)$ or after $(43 \%)$ device utilization [35]. In a randomized study, 30 patients with advanced cancer and persistent dyspnea were assigned to either HFO or NIV (BiPAP levels) for $2 \mathrm{~h}$. Dyspnea was assessed with a numeric rating scale and modified Borg scale before and after the intervention. Vital signs, transcutaneous carbon dioxide and adverse effects were also recorded. Both NIV and HFNT were associated with significant and extended relief of dyspnea and enhanced physiologic parameters without significant adverse effects [36].

\section{Clinical considerations on the use of palliative ventilation and research agenda}

NIV is increasingly used as a palliative strategy when endotracheal ventilation is deemed inappropriate. Palliative ventilation can either be administered to offer a chance for survival, with a resolution of an acute respiratory failure, particularly in hematologic population [19], or to alleviate the symptoms of respiratory distress in dying patients [12]. Patients in the terminal stage could be already receiving NIV at home for pulmonary disease, or have previously given a 'do not intubate/ tracheostomise' order. More frequently, patients with respiratory failure due to the disease or its complications may present suffering from severe dyspnea not respondent to conventional pharmacological therapy. There is also a subgroup of patients, who are temporarily treated with palliative ventilation while waiting for further decisions and appropriate communication, because an abrupt switching from an intensive setting to end of life care [21]. For patients treated on long-term NIV at home, the main goal is to optimize their daily quality of life. NIV settings and interface should be optimized and oxygen should be targeted to obtain and $\mathrm{SaO}_{2}$ around $90 \%$. It is also mandatory to pay particular attention on how the patient tolerates NIV. Sputum retention should be treated with manual or mechanical aids or even with nasal tracheal suctioning. An essential pre-requisite before palliative ventilation is started in a palliative setting is to assess the benefits for the patient, as well as the skills and experience of the care-givers [22]. Palliative ventilation should not cause patients discomfort and oxygen should be targeted according to patient's dyspnea. Alarm should be turned off because they generate distress and may make the family members even more anxious. The real priority is to make sure the patient is comfortable; appropriate sedatives (benzodiazepines) and opioid drugs can be administered if necessary to facilitate patients' compliance for the first hours until stabilization is achieved. Although NIV is considered a 'non aggressive' technique, its use in a palliative care setting still remains debatable [37-39]. HFNC seems to offer advantageous properties, particularly for patient's compliance and acceptability, and should precede NIV application. The pioneer and encouraging studies in palliative care patients near to end of life are limited and often of short duration.

Further studies focused on quality of life and typical palliative care issues should be performed to provide a further option in a difficult clinical context such as respiratory failure with dyspnea. No information exists about the combination with common drugs used for relieving dyspnea such as opioids. The appropriateness of palliative ventilation should be reported in studies that rely more on qualitative criteria (patient comfort, end-of-life process, family burden and health-care provider satisfaction) than quantitative criteria (rate of palliative NIV use and mortality). Many patients and their relatives are potentially interested in ensuring and maximizing comfort in the later stages of the disease. Interestingly, some of them also expressed the desire to maintain cognition and the ability to communicate [40]. However, at this very critical stage, the patient is often no longer able to cooperate. NIV may be uncomfortable and may cause undesirable side-effects such as skin lesions, irritation of the eyes, abdominal bloating and in rare cases barotraumatic events. Thus, any palliative ventilation intervention needs to be discussed with patients, when possible, or relatives. Although palliative ventilation may be beneficial in short-term or long-term conditions, some patients may wish to stop the intervention or simply because palliative ventilation has exhausted his function. More data are necessary to delineate a strategic plan for transition or discontinuation of palliative ventilation. The withdrawal of any form of palliative ventilation appears to pose considerable challenges to palliative physicians for the emotional, practical and ethical implications [41]. Ethical and legal rights to withdrawal from treatment, discussions with family and colleagues, experiences of legal advice and issues contributing to ethical complexity are the most challenging issues to afford [42].

Conflict of interest The authors declare that they have no conflict of interest.

Disclosure Authors have full control of all primary data. We do not use clinical data to be eventually reviewed by the journal. 


\section{References}

1. Mahler DA, O’Donnell DE (2014). In "Dyspnea. Mechanisms, measurement, and management". CRC Press, Boca Raton

2. Thomas JR, von Gunten CF (2002) Clinical management of dyspnoea. Lancet Oncol 3:223-228

3. Hauser CA, Stockler MR, Tattersall MHP (2006) Rognostic factors in patients with recently diagnosed incurable cancer: a systematic review. Support Care Cancer 14:999-1011

4. Reddy SK, Parsons HA, Elsayem A, Palmer JL, Bruera E (2009) Characteristics and correlates of dyspnea in patients with advanced cancer. J Palliat Med 12:29-36

5. Mercadante S, Casuccio A, Fulfaro F (2000) The course of symptom frequency and intensity in advanced cancer patients followed at home. J Pain Symptom Manag 20:104-112

6. Mercadante S, Porzio G, Valle A et al (2012) Emergencies in patients with advanced cancer followed at home. J Pain Symptom Manag 44:295-300

7. Mercadante S, Masedu F, Valenti M, Mercadante A, Aielli F (2016) The characteristics of advanced cancer patients followed at home, but admitted to the hospital for the last days of life. Intern Emerg Med 11:713-718

8. Mercadante S, Porzio G, Valle A, Aielli F, Casuccio A (2014) Palliative sedation in patients with advanced cancer followed at home: a prospective study. J Pain Symptom Manag 47:860-866

9. Barnes H, McDonald J, Smallwood N, Manser R (2016) Opioids for the palliation of refractory breathlessness in adults with advanced disease and terminal illness. Cochrane Database Syst Rev 31(3):CD011008

10. Abernethy AP, McDonald CF, Frith PA et al (2010) Effect of palliative oxygen versus room air in relief of breathlessness in patients with refractory dyspnoea: a double-blind, randomised controlled trial. Lancet 4(376):784-793

11. Nava S, Cuomo AM (2004) Acute respiratory failure in the cancer patient: the role of non-invasive mechanical ventilation. Crit Rev Oncol Hematol 51:91-103

12. Azoulay E, Demoule A, Jaber S et al (2011) Palliative noninvasive ventilation in patients with acute respiratory failure. Intensive Care Med 37:1250-1257

13. Gregoretti C, Mattei A, Carlucci A. (2015) Noninvasive Ventilation Outside the Critical Care Unit. In "Practical Issues Updates in Anesthesia and Intensive Care". D. Chiumello (ed.), Springer International Publishing Switzerland 139-47

14. Boldrini R, Fasano L, Nava S (2012) Noninvasive mechanical ventilation. Curr Opin Crit Care 18:48-53

15. Nava S, Hill N (2009) Non-invasive ventilation in acute respiratory failure. Lancet 18(374):250-259

16. Elliott MW, Confalonieri M, Nava S (2002) Where to perform noninvasive ventilation? Eur Respir J 19:1159-1166

17. Landoni G, Zangrillo A, Cabrini L (2012) Non-invasive ventilation outside the ICU. In: Louis J (ed) Annual update of intensive care and emergency medicine. Vincent. Springer-Verlag, Berlin, pp 207-218

18. Cabrini L, Antonelli M, Savoia G, Landriscina M (2011) Noninvasive ventilation outside of the intensive care unit: an Italian survey. Minerva Anestesiol 77:313-322

19. Nava S, Mercadante S. (2011) NIV Outside the ICU. In" Pulmonary Involvement in Patients with Hematological Malignancies, Azoulay E. ed, Springer-Verlag Berlin Heidelberg 623-9

20. Nava S, Hill N (2009) Non-invasive ventilation in acute respiratory failure. Lancet 374:250-259

21. Mercadante S, Villari P, David F, Agozzino C (2009) Noninvasive ventilation for the treatment of dyspnea as a bridge from intensive to end-of-life care. J Pain Symptom Manag 38:e5-e7
22. Perrin C, Jullien V, Duval Y, Defrance C (2008) Noninvasive ventilation in palliative care and near the end of life. Rev Mal Respir 25: $1227-1236$

23. Nagata K, Morimoto T, Fujimoto D et al (2015) Efficacy of highflow nasal cannula therapy in acute hypoxemic respiratory failure: decreased use of mechanical ventilation. Respir Care 60:1390 1396

24. Cirio S, Piran M, Vitacca M et al (2016) Effects of heated and humidified high flow gases during high-intensity constant-load exercise on severe COPD patients with ventilatory limitation. Respir Med 118:128-132

25. Peters SG, Holets SR, Gay PC (2013) High-flow nasal cannula therapy in do-not-intubate patients with hypoxemic respiratory distress. Respir Care:58-54

26. Chanques G, Constantin JM, Sauter M et al (2009) Discomfort associated with under humidified high-flow oxygen therapy in critically ill patients. Intensive Care Med 35:996-1003

27. Maggiore SM, Idone FA, Vaschetto R et al (2014) Nasal high-flow versus Venturi mask oxygen therapy after extubation. Effects on oxygenation, comfort, and clinical outcome. Am J Respir Crit Care Med 190:282-288

28. Dysart K, Miller TL, Wolfson MR, Shaffer TH (2009) Research in high flow therapy: mechanisms of action. Respir Med 103:1400 1405

29. Mündel T, Feng S, Tatkov S, Schneider H (2013) Mechanisms of nasal high flow on ventilation during wakefulness and sleep. J Appl Physiol 114:1058-1065

30. Möller W, Celik G, Feng S (2015) J Appl Physiol 11:1525-1532

31. Elliott MW. (2011) Non-invasive ventilation: established and expanding roles. Clin Med (Lond) 11:150-153

32. Cuomo A, Delmastro M, Ceriana P (2004) Noninvasive mechanical ventilation as a palliative treatment of acute respiratory failure in patients with end-stage solid cancer. Palliat Med 18: 602-610

33. Nava S, Ferrer M, Esquinas A et al (2013) Palliative use of non-invasive ventilation in end-of-life patients with solid tumours: a randomised feasibility trial. Lancet Oncol 14:219227

34. Peters SG, Holets SR, Gay PC (2013) High-flow nasal cannula therapy in do-not-intubate patients with hypoxemic respiratory distress. Respir Care 58:597-600

35. Epstein AS, Hartridge-Lambert SK, Ramaker JS, Voigt LP, Portlock CS (2011) Humidified high-flow nasal oxygen utilization in patients with cancer at Memorial Sloan-Kettering Cancer Center. J Palliat Med 14:835-839

36. Hui D, Morgado M, Chisholm G et al (2013) High flow oxygen and bilevel positive airway pressure for persistent dyspnea in patients with advanced cancer: a phase II randomized trial. J Pain Symptom Manag 46:463-473

37. Gregoretti C, Confalonieri M, Navalesi P et al (2002) Evaluation of patient skin breakdown and comfort with a new face mask for noninvasive ventilation: a multi-center study. Intensive Care Med 28: 278-284

38. Selecky PA, Eliasson CA, Hall RI, American College of Chest Physicians et al (2005) Palliative and end-of-life care for patients with cardiopulmonary diseases: American College of Chest Physicians position statement. Chest 128: 3599-3610

39. Perrin C, Jullien V, Lemoigne F (2004) Practical and technical aspects of noninvasive ventilation. Rev Mal Respir 21:556-566

40. Curtis JR, Cook DJ, Sinuff T, Society of Critical Care Medicine Palliative Noninvasive Positive VentilationTask Force et al (2007) Noninvasive positive pressure ventilation in critical and palliative care settings: understanding the goals of therapy. Crit Care Med 35: 932-939 
41. Faull C, Rowe Haynes C, Oliver D (2004) Issues for palliative medicine doctors surrounding the withdrawal of noninvasive ventilation at the request of a patient with motor neurone disease: a scoping study. BMJ Support Palliat Care 4:43-49
42. Phelps K, Regen E, Oliver D, McDermott C, Faull C. (2015) Withdrawal of ventilation at the patient's request in MND: a retrospective exploration of the ethical and legal issues that have arisen for doctors in the UK. BMJ Support Palliat Care Sep 11. doi: 10.1136/bmjspcare-2014-000826 